\title{
High-throughput optical wireless solutions for intra-satellite communications
}

E. Ertunc, G. Cossu, A. Messa, E. Ciaramella

E. Ertunc, G. Cossu, A. Messa, E. Ciaramella, "High-throughput optical wireless solutions for intra-satellite communications," Proc. SPIE 11852, International Conference on Space Optics - ICSO 2020, 118525 U (11 June 2021); doi: 10.1117/12.2600015

SPIE Event: International Conference on Space Optics - ICSO 2021, 2021, Online Only 


\section{International Conference on Space Optics-ICSO 2020}

Virtual Conference

30 March-2 April 2021

Edited by Bruno Cugny, Zoran Sodnik, and Nikos Karafolas
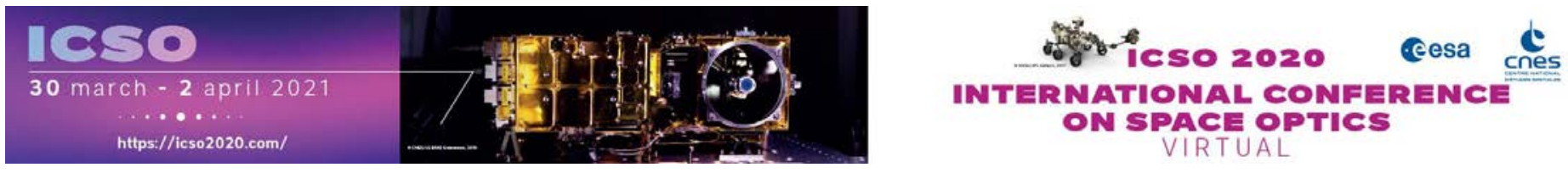

\section{High-throughput optical wireless solutions for intra-satellite communications}

\section{Cesa issopreatings denes}




\title{
High-Throughput Optical Wireless Solution for Intra-Satellite Communications
}

\author{
E. Ertunc*a, G. Cossu ${ }^{\text {a }}$ A. Messa ${ }^{\text {a }}$, E. Ciaramella ${ }^{\mathrm{a}}$ \\ 'Scuola Superiore Sant'Anna, Via G. Moruzzi, 1, 56124 Pisa (Italy)
}

\begin{abstract}
We theoretically and experimentally demonstrate a $100 \mathrm{Mb} / \mathrm{s}$ Optical Wireless Communication (OWC) system for 3U (30x30x30 centimeter) intra-CubeSat. The system has low power consumption and is made of low cost commercially available off-the-shelf (COTS) components such as VCSEL, APD, Si-PD and common ball lenses. Since the system is for the $3 \mathrm{U}$ CubeSat, $40 \mathrm{~cm}$ distance between optical transceiver was targeted. We successfully designed the system and tested with $4 \mathrm{~mm}$ and $5 \mathrm{~mm}$ ball lenses to optimize our system for the future activities.
\end{abstract}

Keywords: Optical wireless communications, laser communications, free space, satellite communication, CubeSat

\section{INTRODUCTION}

Since the last decade, small spacecrafts $(\mathrm{S} / \mathrm{C})$ are gaining a dominant role in navigation services, scientific missions, and TV broadcasting in both the military and the civil sectors. There are several types of small satellites, which are usually classified according to their mass: mini (100-500 kg), micro (10-100 kg), nano (1-10 kg), pico (1 kg), femto (<100 gr) [1]. Recently, applications of CubeSats nanosatellites are significantly growing in the field of academic, government, defense and commercial research. The CubeSat design is based on modular multiple units (NU) starting from the basic element, i.e. $1 \mathrm{U}$ that has dimension of $10 \times 10 \times 10$ centimeter with a mass of fewer than 1.33 kilograms. CubeSats are located in low earth orbit (LEO). The smallest is $0.25 \mathrm{U}$ and the largest is $12 \mathrm{U}$ [2]. The market of CubeSat is envisaged to grow gradually from USD 152 million in 2018 to USD 375 million by 2023, at a CAGR of 19.87\% from 2018 to 2023 [3].

Today, the advent of the digital revolution and the thriving of countless services and data-hungry applications, the amount of data to be transmitted wireless is significantly increasing, RF and microwave systems show their intrinsic capacity limits, and this is no longer sustainable. The required volumes of data generated by CubeSat so far have been transmitted using the radio frequency (RF) or microwave bands to ground [4].

Optical wireless communication (OWC) is a promising alternative to RF legacy satellite links since it can support very high rates by means of commercially available off-the-shelf (COTS) components and it can also exploit a large unregulated portion of the spectrum. OWC does not suffer nor produce EMI. Shortly soon, most of the foreseen OWC solutions are related to ground-to-satellite downlink, inter-satellite and deep-space communications. However, OWC can be conveniently considered for intra-satellite communications. Especially when low-cost, high bitrate and lower mass are needed, OWC allows removing expensive and bulky cables. CubeSat and very small satellites are a clear example of these application scenarios.

A key feature of the OWC systems is robustness: the systems must be resilient to misalignment and work with partial shadowing, to be compatible with the considered application scenario, where vibrations and partial obstructions can occur. To this aim, we measured the bit error rate (BER) as a function of the radial misalignment in direct line of sight (d-LoS) configurations and we repeated the same measurement inserting an opaque body on the line of sight to partially shadow the transmission. As CubeSats are very tiny satellites, point-to-point communication system is very suitable. Since the aim is to fully exploit from The OWC system, d-LoS architecture is used for intra-CubeSat communication as shown in Fig 1.

*ezgi.ertunc@santannapisa.it;

https://www.santannapisa.it/en/ 


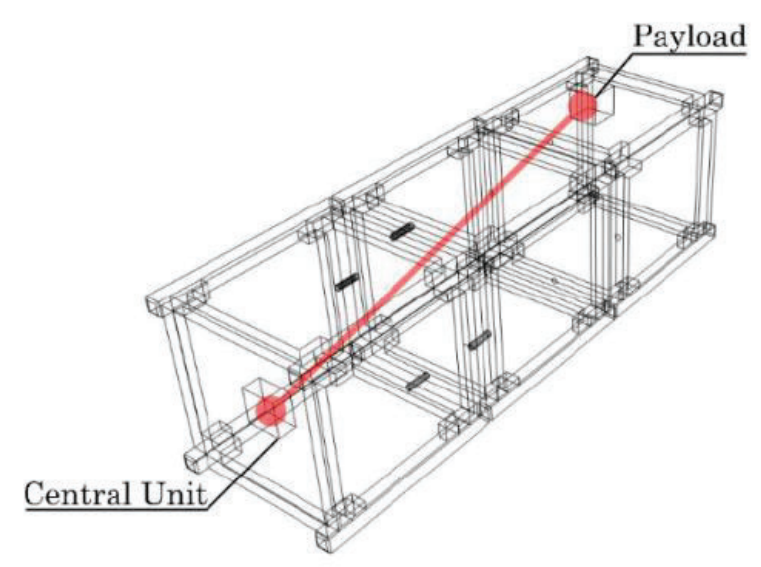

Fig. 1 CubeSat scenario with VLC system, enabling high bit rate transmission between payload and central unit.

There are some preliminary works on OWC systems for intra-satellite communication applications using light emitting diodes (LEDs) [5-8]. Although, the studies indicate low bandwidth and high power, LEDs are not suitable for reaching higher bandwidth and low power consumption. Therefore, recently, we proposed a new approach of OWC system for satellite communication [9-10] using COTS components having low-cost and low-power consumption. The system leverages on the most recent achievements for indoor OWC.

In this work, we present a design study on a low-cost and low-power visible light communication (VLC) link, emulating a communication between two units inside a small satellite (CubeSat 3U). Our point-to-point VLC system works at 100 $\mathrm{Mbit} / \mathrm{s}$ reaching up to $40 \mathrm{~cm}$ distance. The OWC link uses COTS components. On the transmitter (TX) side, we use a 670 $\mathrm{nm}$ vertical cavity surface-emitting laser (VCSEL) with a ball lens to obtain the proper divergence; the VCSEL is chosen thanks to its wide bandwidth, low power consumption and low cost. On the receiver (RX) side, we choose an avalanche photodiode (APD) and Si based photodiode (Si-PD). The structure of this paper is as follows: first, the optical components, sensitivity of the system and simulation are explained in the system design part. Then, system setup and results are presented. The paper is finalized with the conclusion.

\section{SYSTEM DESIGN}

\section{Optical Components}

The requirements of the CubeSat applications are very tight, due to lack of space and power consumptions. Since we are assuming the distance between TX and RX to be $40 \mathrm{~cm}$, the active area of RX and tolerance to misalignment should be a minimum of $\sim 6 \mathrm{~mm}$. The beam emission angle is calculated by simple math and it is a minimum of $4^{\circ}$.

In our OWC system, on the TX side, we used a $670 \mathrm{~nm}$ VCSEL thanks to its features; it has $1 \mathrm{GHz}$ electrical bandwidth, $1 \mathrm{~mW}$ output power and its beam intensity distribution has good circular symmetry. VCSELs require a lower electrical drive current (even lower than LEDs), and this has a positive impact on figures like power consumption, efficiency, and longer lifespan. This comes at the cost of a sensibly lower emitted optical power. A ball lens was chosen to increase the concentration of the light on the RX.

At the RX side, we had an AC-coupled APD (bandwidth of $280 \mathrm{MHz}$ active area of $3.14 \mathrm{~mm}^{2}$ ) with an embedded TransImpedance Amplifier (TIA) to observe the quality of the optical signal. Since the APD is AC-coupled, we used an active area of $0.8 \mathrm{~mm}^{2} \mathrm{DC}$-coupled Si-PD to measure the optical power. A $675 \mathrm{~nm} \pm 100 \mathrm{~nm}$ bandpass filter was placed in front of the DC-coupled Si-PD to avoid ambient light since the spectral response range of the Si-PD is 200-1100 nm. In order to measure the sensitivity of the system, variable optical attenuators were used to reduce the optical power.

\section{Sensitivity of the Receiver}

First, we experimentally measured the link sensitivity. In order to obtain higher received optical power $\left(\mathrm{P}_{\mathrm{RX}}\right)$, both TX and RX were alligned as face-to-face at the link distance of $40 \mathrm{~cm}$. The peak-to-peak voltage level was adjusted on the pulse 
pattern generator at $1 \mathrm{~V}$. The optical power was varied by the optical attenuators and BER measurements were performed. We observed BER $<10^{-9}$ without any Forward Error Correction (FEC) at power of $>-33 \mathrm{dBm}$.

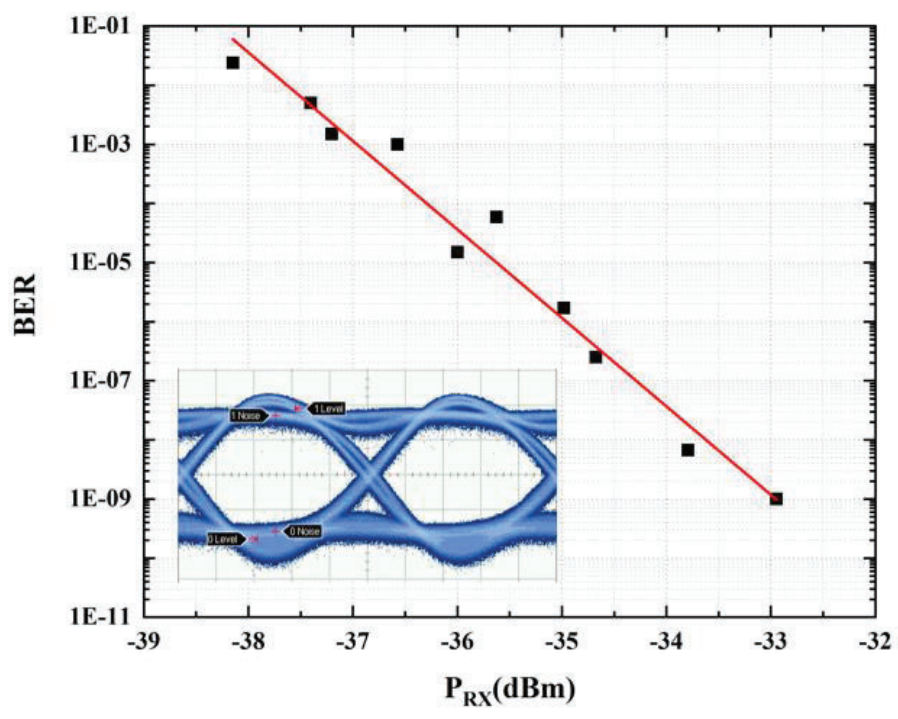

Fig. 2 the eye diagram of $100 \mathrm{Mb} / \mathrm{s}$ transmission at the align position and the link sensitivity (red curve is the reference line)

\section{Simulation}

First, we performed the simulation of the OWC system by using the TracePro (ray-tracing) software, assuming the real parameters of the optical components. The aim of the simulation is to optimize the distance (d) between TX and the ball lens for the OWC system and optimize the system vs the radial misalignment. The considered link distance reaches up to $40 \mathrm{~cm}$. According to the simulation as shown in Fig. 3, we reached $\pm 17.4 \mathrm{~mm}$ radial misalignment for $4 \mathrm{~mm}$ ball lens $(\mathrm{d}=4.3 \mathrm{~mm})$ and $\pm 16.7 \mathrm{~mm}$ radial misalignment for $5 \mathrm{~mm}$ ball lens $(\mathrm{d}=6.5)$.

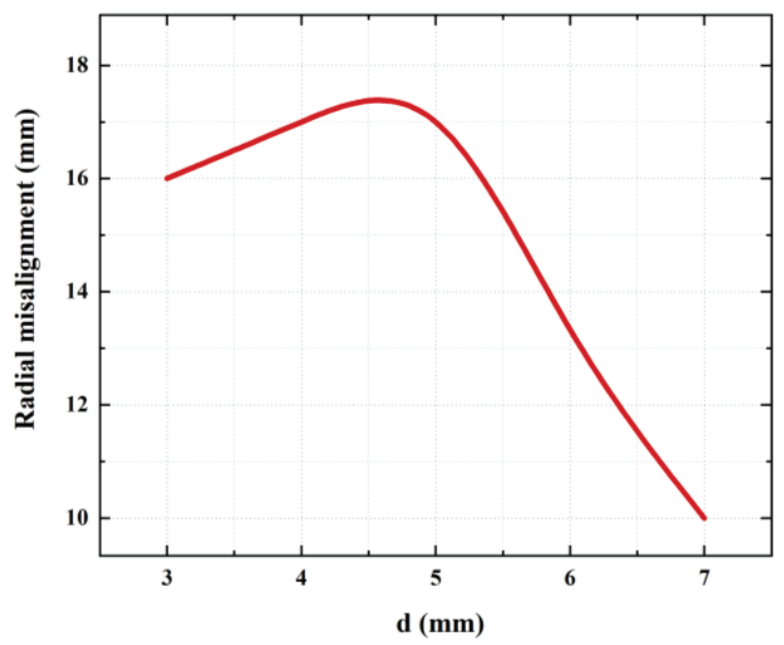

(a)

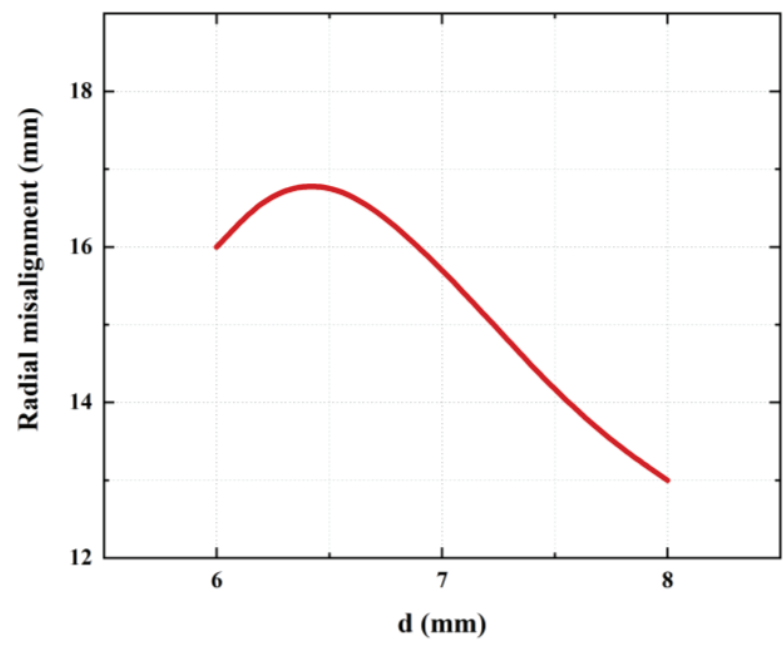

(b)

Fig. 3 Simulation results for $4 \mathrm{~mm}(\mathrm{a})$, and $5 \mathrm{~mm}(\mathrm{~b})$ ball lens 


\section{EXPERIMENTAL SETUP AND RESULTS}

When designing high-speed OWC systems, various features must be taken into account. The key foundation of any OWC system is the link design and the proper choice between directed line-of-sight (d-LoS) and non-line-of-sight (n-LoS). Reflections do not need to be taken into consideration for d-LoS; therefore, the beam emission angle of the transmitter can be calculated easily. The key features of d-LoS are the concentrated beam, high level of directivity of the optical signal, lower effect of ambient light and higher signal-to-noise ratio (SNR) value. Based on the features of VCSEL and a ball lens, the d-LoS configuration will be fully utilized.

In the OWC setup, as shown in Fig. 4, the $670 \mathrm{~nm}$ VCSEL was directly modulated at $100 \mathrm{Mb} / \mathrm{s}$ by a Pseudo-Random Bit Sequence (PRBS) Non-Return-to-Zero (NRZ) sequence (length of $2^{31}-1$ bits), using the pulse pattern generator (PPG). The bias current of the VCSEL was set at $2.4 \mathrm{~mA}$ and the electrical data had $1 \mathrm{~V}$ amplitude. The light travels in free space afterwards, the output signal was connected to the Signal Quality Analyzer (SQA) for the optical signal analysis and the BER Tester (BERT) for the BER measurements.

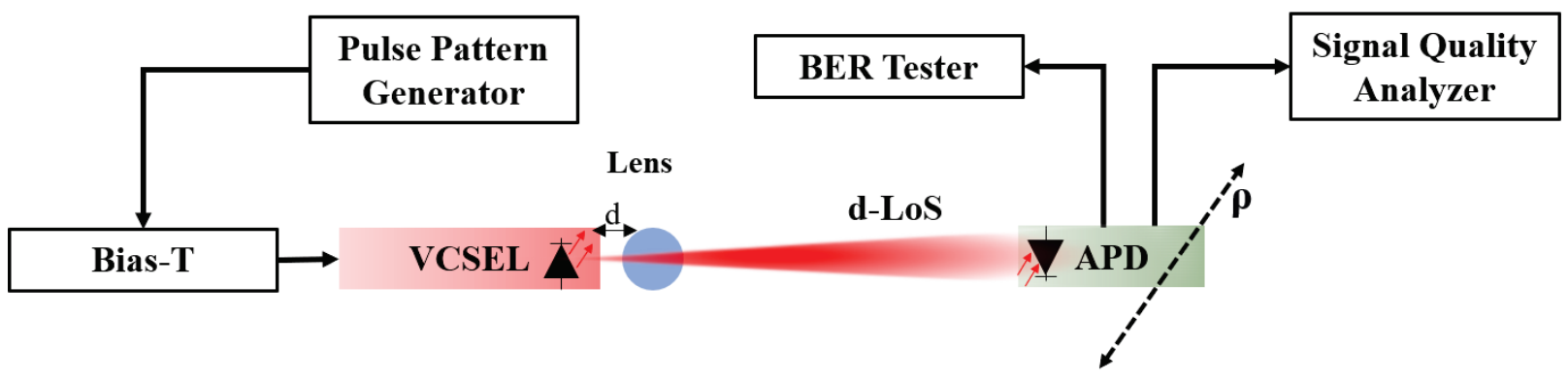

Fig. 4 Setup of the test of the OWC system

The BER measurements were performed using the TX with ball lenses of of $4 \mathrm{~mm}$ and $5 \mathrm{~mm}$ diameter to realize the best condition for $3 \mathrm{U}$ CubeSat in terms of signal quality, displacement values and $\mathrm{P}_{\mathrm{RX}}$. The ball lenses were placed in front of the TX to collimate and obtain a well concentrated beam.

In optical communication systems, the signal quality is estimated by Q-factor which is expressed by 'ones' and 'zeros' of the eye diagram. It is an accurate way to estimate the system margin theoretically and it is expressed as given by:

$$
\mathrm{Q}=\frac{\mu_{1}-\mu_{0}}{\sigma_{1}+\sigma_{0}}
$$

In eq. $3.1 \mu_{1}$ and $\mu_{0}$ are the mean value, $\sigma_{1}$ and $\sigma_{0}$ are the standard deviation of the Gaussian noise. According to the space application, the BER of $<10^{-9}$ was projected . There is a strong relationship between Q factor and BER. Thus, we can easily estimate the BER values by utilizing the equation by:

$$
\operatorname{BER}=\frac{1}{2} \operatorname{erfc}\left(\frac{\mathrm{Q}}{\sqrt{2}}\right)
$$

We characterized the system with the $4 \mathrm{~mm}$ ball lens and $5 \mathrm{~mm}$ ball lens at the same condition as sensitivity measurement (alligned position as $\rho=0$ ). The wide opening eye diagrams at $100 \mathrm{Mb} / \mathrm{s}$ are indicated in Fig $5(\mathrm{a}-\mathrm{b})$. The time scale is 5 $\mathrm{ns} / \mathrm{div}$ for both eye diagrams. Amplitude of the signal is $1 \mathrm{~V}$ and $1.3 \mathrm{~V}$ for Fig. 5 (a) and (b), respectively. 


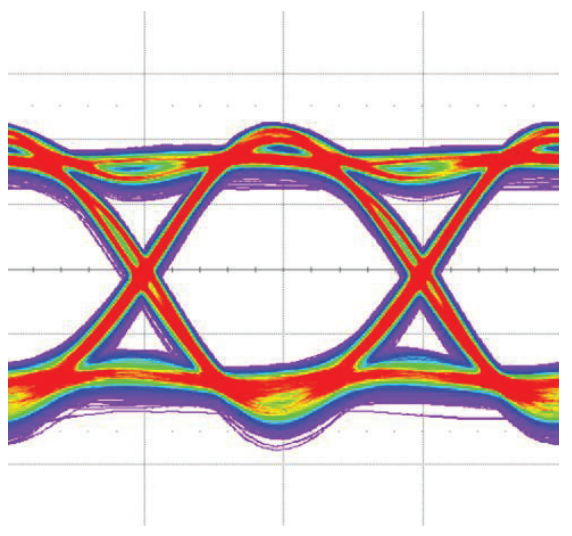

(a) $4 \mathrm{~mm}$ ball lens

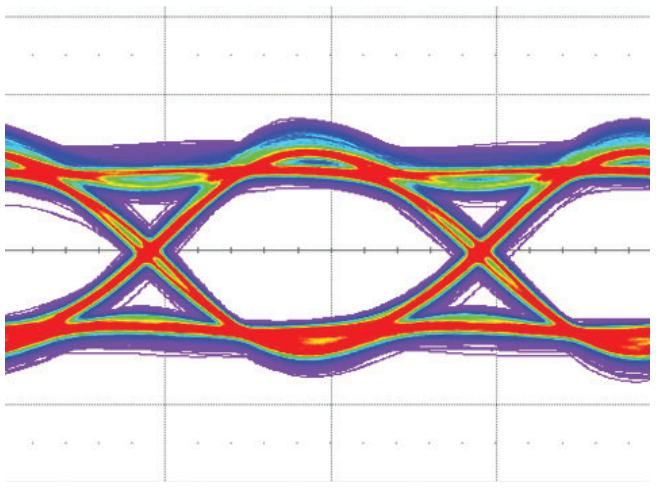

(b) $5 \mathrm{~mm}$ ball lens

Fig. 5 Eye diagram of the OWC system by using $4 \mathrm{~mm}$ ball lens (a), and $5 \mathrm{~mm}$ ball lens (b)

Tolerance to misalignment measurements were performed in the same condition with $4 \mathrm{~mm}$ and $5 \mathrm{~mm}$ ball lenses. The misalignment measurement was continued until the signal was completely lost $\left(\mathrm{BER}>10^{-2}\right)$.

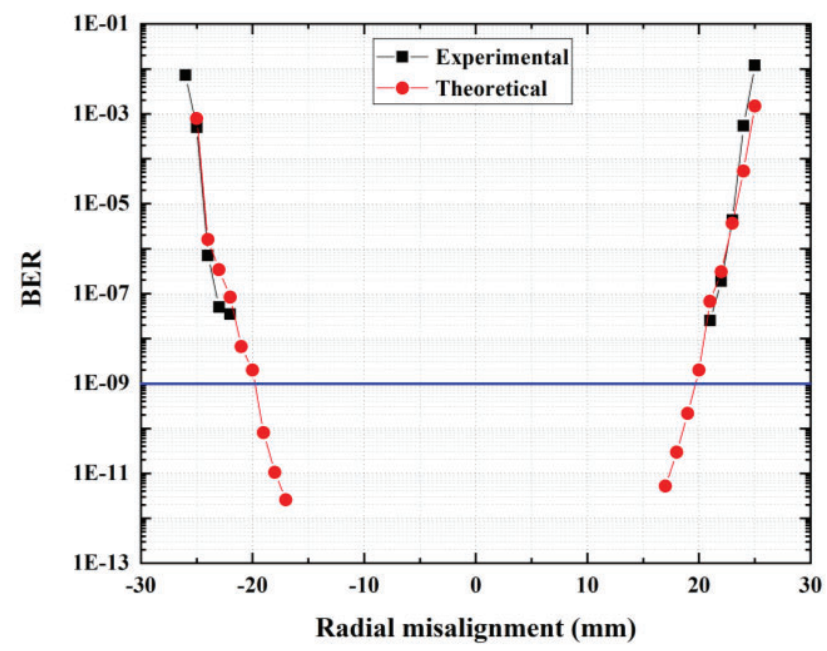

(a)

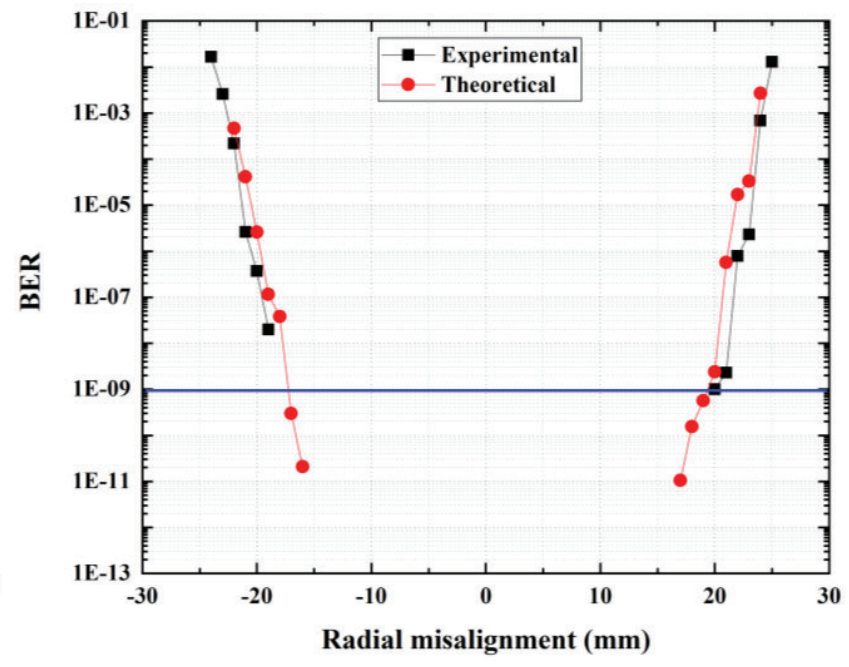

(b)

Fig. 6 BER as a function of radial displacement using $4 \mathrm{~mm}$ ball lens (a) and $5 \mathrm{~mm}$ ball lens (b)

Fig. 6 (a-b) reports the BER values as a function of radial misalignment for the two ball lenses. They indicate the tolerance to misalignment. The blue line indicates the tolerance limitation at $B E R=10^{-9}$. For the higher accuracy results, we compared the estimated BER results by exploiting the Q-factor. Eventually, the theoretical results and experimental results are quite similar. Therefore, in practical experiments the Q-factor allows to find out the accurate results in a very short time.

As seen from Fig. 6 (a-b), we observe slightly better tolerance to misalignment with $4 \mathrm{~mm}$ ball lens $\pm 2.1 \mathrm{~cm}$ rather than with $5 \mathrm{~mm}$ ball $\pm 1.9 \mathrm{~cm}$. 


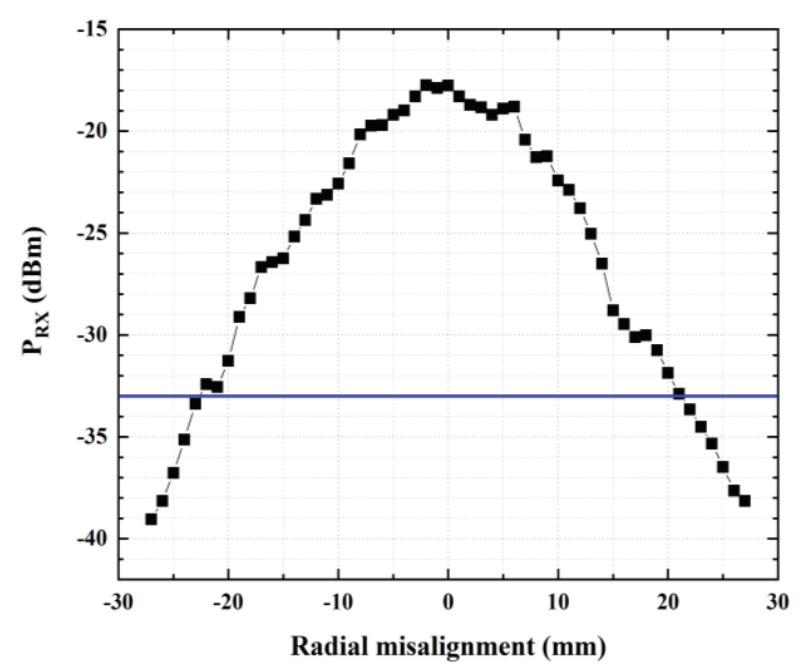

(a)

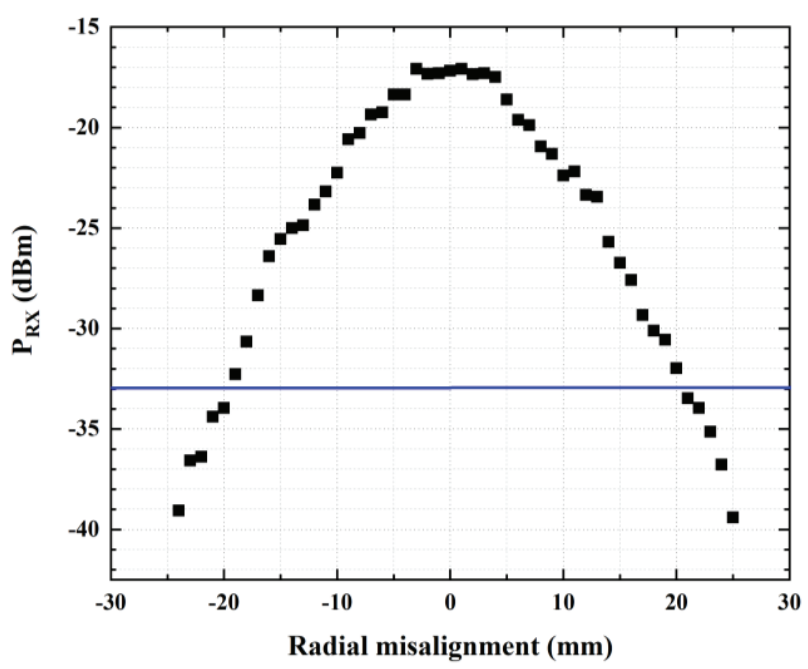

(b)

Fig 7. $\mathrm{P}_{\mathrm{RX}}$ as a function of radial misalignment using (a) $4 \mathrm{~mm}$ ball lens and (b) $5 \mathrm{~mm}$ ball lens

According to the results that are shown in Fig $7(a-b)$, when the RX at the origin $(\rho=0)$, we obtain optical power of $\sim 18$ and $\sim 17 \mathrm{dBm}$, respectively. The blue line indicates the limit of the safe region where BER $\leq 10^{-9}$ where the $P_{R X}$ is $-33 \mathrm{dBm}$. The system with the $4 \mathrm{~mm}$ ball lens has slightly higher tolerance to misalignment.

\section{CONCLUSION}

In this paper, we experimentally and theoretically presented preliminary results using our OWC system for a $3 \mathrm{U}$ CubeSat reaching $100 \mathrm{Mb} / \mathrm{s}$ at $40 \mathrm{~cm}$ distance. We used a common on-off keying (OOK) signal. The misalignment tolerance was measured to be $\pm 20 \mathrm{~mm}$ using $4 \mathrm{~mm}$ ball lens and $5 \mathrm{~mm}$ ball lens by targeting the BER of $10^{-9}$ without any FEC. It does not need a complex alignment in the satellite based on the system was based on COTS components such as $670 \mathrm{~nm}$ VCSEL, APD, Si-PD and ball lenses. This preliminary study allows to estimate the requirements of our OWC system for current rate and estimate its potential for future works of intra-satellite communications. Future work will also be aimed to minimize the weight of the system and packaging of the optical devices.

\section{ACKNOWLEDGMENT}

This work was partly supported by the Italian Space Agency (ASI) under project Free Space Optical Communications for Space Applications (FOCS).

\section{REFERENCES}

[1] Mission Design Division Stuff, "Small Spacecraft Technology State of Art," Ames Research Center, NASA/TP-2015216648/REV1, Moffett Field, California 94035-1000, 2015.

[2] E. Kulu, "Nanosats Database,". www.nanosats.eu 
[3] A. Gulhane, Markets and Markets, "CubeSat Market by Application (Earth Observation \& Traffic Monitoring, Communication, Science \& Technology and Education), End User (Government \& Military, Commercial, Non-profit Organizations), Size, Subsystem, and Region - Global Forecast to 2023", URL: https://www.marketsandmarkets.com/Market-Reports/cubesat-market-58068326.html,

[4] N. Saeed, A. Elzanaty, H. Almorad, H. Dahrouj, T. Y. Al-Naffouri and M. -S. Alouini, "CubeSat Communications: Recent Advances and Future Challenges," in IEEE Communications Surveys \& Tutorials, vol. 22, no. 3, pp. 18391862, thirdquarter 2020, doi: 10.1109/COMST.2020.2990499.

[5] L. Wood, W. Ivancic and K. Dörpelkus, "Using Light-Emitting Diodes for intersatellite links," 2010 IEEE Aerospace Conference, Big Sky, MT, USA, 2010, pp. 1-6, doi: 10.1109/AERO.2010.5446711.

[6] A. Anzagira and W. Edmonson, "Non-Orthogonal Multiple Access (NOMA) for LED-based Visible Light InterSatellite Communications," 2018 6th IEEE International Conference on Wireless for Space and Extreme Environments (WiSEE), Huntsville, AL, USA, 2018, pp. 24-29, doi: 10.1109/WiSEE.2018.8637346.

[7] D. N. Amanor, W. W. Edmonson and F. Afghah, "Intersatellite Communication System Based on Visible Light," in IEEE Transactions on Aerospace and Electronic Systems, vol. 54, no. 6, pp. 2888-2899, Dec. 2018, doi: 10.1109/TAES.2018.2832938.

[8] D. N. Amanor, W. W. Edmonson and F. Afghah, "Link performance improvement via design variables optimization in LED-based VLC system for inter-satellite communication," 2017 IEEE International Conference on Wireless for Space and Extreme Environments (WiSEE), Montreal, QC, 2017, pp. 7-12, doi: 10.1109/WiSEE.2017.8124884.

[9] E. Ciaramella et al., "TOWS: Introducing Optical Wireless for Satellites," 2019 21st International Conference on Transparent Optical Networks (ICTON), Angers, France, 2019, pp. 1-4, doi: 10.1109/ICTON.2019.8840565.

[10]E. Ciaramella et al., "Prospects of Visible Light Communications in Satellites," 2020 22nd International Conference on Transparent Optical Networks (ICTON), Bari, Italy, 2020, pp. 1-4, doi: 10.1109/ICTON51198.2020.9203541. 